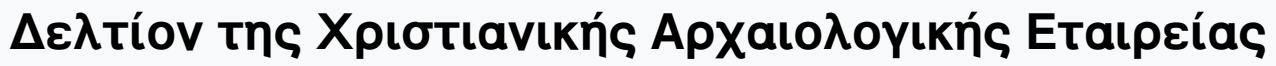

Tóp. 4 (1966)

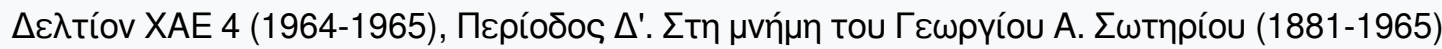

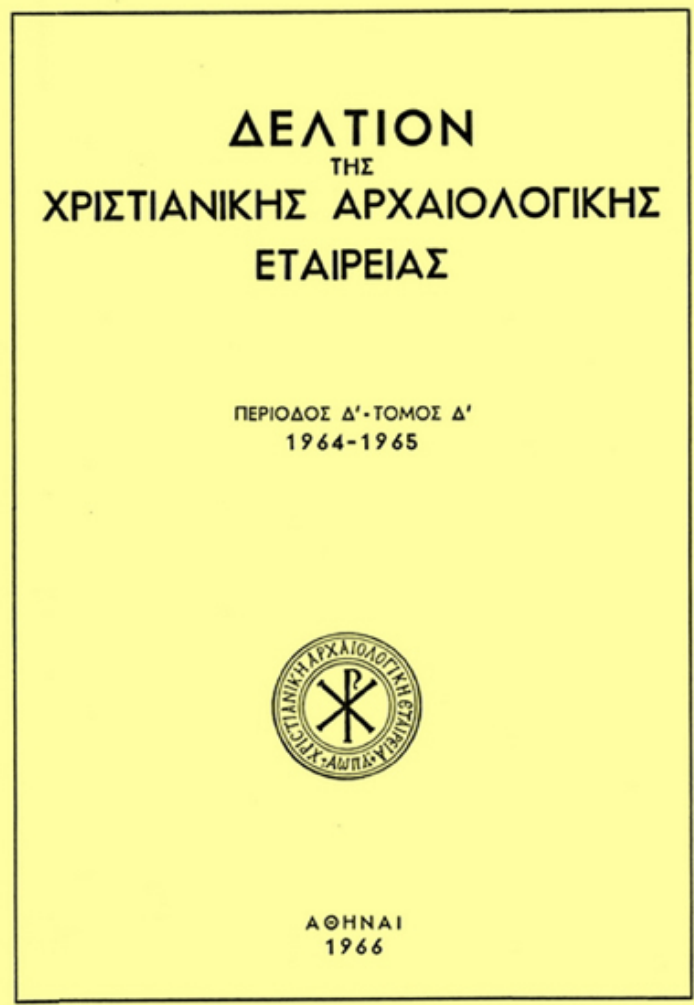

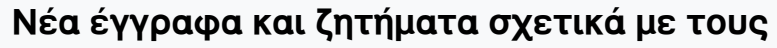

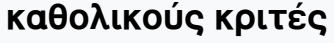

PaUl LEMERLE

doi: $10.12681 /$ dchae.753 


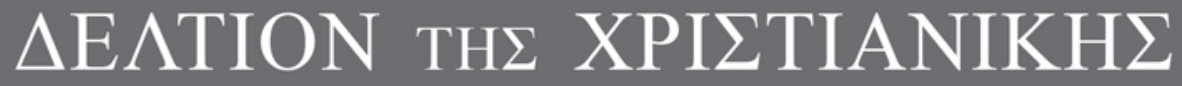 APXAIO $\Lambda$ OГIKH $\Sigma$ ETAIPEIA $\Sigma$}

Documents et problèmes nouveaux concernant les Juges généraux

Paul LEMERLE

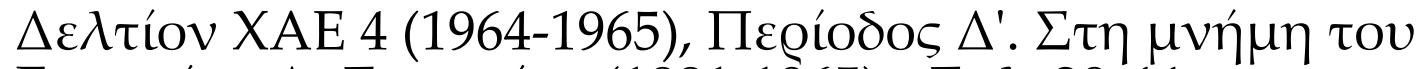

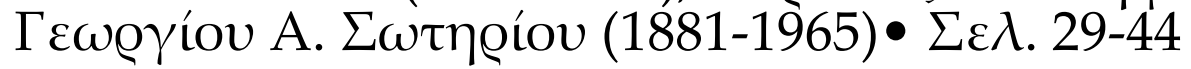

A@HNA 1966 


\section{DOCUMENTS ET PROBLÈMES NOUVEAUX CONCERNANT LES JUGES GÉNÉRAUX}

J'ai eu l'occasion de publier, il y aura bientôt quinze ans, les résultats de quelques recherhes sur les institutions judiciaires à l'époque des Paléologues, et en particulier sur le Juge général des Grecs ${ }^{1}$. Ces articles semblent avoir attiré l'attention sur un titre et une fonction auxquels on n'en avait guère prêté jusque là : divers documents, inédits il y a quinze ans, ont été depuis signalés ou édités. J'ai moi-même, d'autre part, fait quelques observations nouvelles. Je voudrais, ici, rassembler ces données, pour examiner si elles se laissent intégrer dans le tableau que j'avais cru naguère pouvoir tracer, et quèls compléments ou retouches elles conduisent à y apporter.

Je rappelle d'abord, dans les grandes lignes, ce qui semblait acquis. Le tribunal impérial, tribunal suprême de l'empire, reconstitué probablement par Michel VIII après la fin de la domination latine, puis transformé en 1296 par Andronic II en un collège souverain de douze juges ecclésiastiques et laïques ${ }^{2}$, reçut enfin d'Andronic III, par des actes promulgués en $1329-1334^{3}$, la forme qu'il devait en gros conserver jusqu'à la chute de Byzance : un collège de "Juges généraux » revêtus des pleins pouvoirs judiciaires. Nous connaissons les quatre membres du premier collège, deux ecclésiastiques et deux laïcs : Joseph métropolite d'Aprô, le diacre et dikaiophylax Grégoire Kleidas, le grand

1. "Le Juge général des Grecs et la réforme judiciaire d'Andronic III" dans Mémorial Louis Petit, Bucarest-Paris, 1948, p. 292-316: "Recherches sur les institutions judiciaires à l'époque des Paléologues, I, Le tribunal impérial ", dans Annuaire de l'Institut de Philologie et d'Histoire orientales et slaves, 9, Bruxelles, 1949 (=Mélanges Henri Grégoire, I), p. $369-384$ (La seconde partie, "Le tribunal du patriarcat ou tribunal synodal ", a paru dans Analecta Bollandiana, $68=$ Mélanges Paul Peeters, II, 1950, p. $318-333$ ); " Note sur la carrière judi-

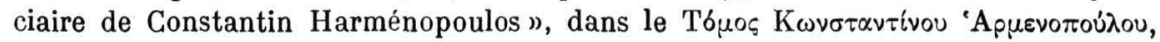
Thessalonique, 1951, p. $243-249$.

2. Cf. maintenant R.K.O.R. (=F. D ölg e r, Regesten der Kaiserurkunden des Oströmischen Reiches, 4. Teil, Regesten von 1282-1341, Munich, 1960), no $2183,2188$.

3. G. maintenant R.K.O.R. no 2743, 2747, 2749, 2805, 2806, 2817. 
dioecète Glavas, et Nicolas Matarangos. Un grand procès de corruption, jugé en 1337 (ou 1336 ?), aboutit à la destitution des trois premiers : seul Matarangos fut reconnu innocent. L'institution n'en fut pourtant pas ébranlée, puisqu'on la voit subsister jusqu'à la fin de l'empire. Mais au cours d'une évolution plus que séculaire, elle subit des changements, encore insuffisamment connus, affectant la composition des collèges (nous en connaissons qui ne comprennent que trois membres, dont un seul d'Église), le fonctionnement du tribunal (à la stricte collégialité des débuts succède un régime où un seul des Juges généraux, par exemple en mission en province, décide et signe), sa souveraineté (illimitée au départ, elle semble avoir été un moment limitée par la compétence du logothète général). Surtout, on voit l'institution se diversifier. Les “Juges généraux des Grecs », qui forment par excellence le tribunal impérial de la capitale, sont attestés jusqu'au bout, puisque Georges Scholarios en fut un. Mais on voit apparaitre des Juges généraux hors du territoire soumis au basileus (et c'est un hommage au succès et aux mérites de l'institution): dans l'empire serbe, dans l'empire de Trébizonde. On assiste aussi, évidemment sous la pression des évènements, à une sorte de décentralisation, qui se manifeste par l'apparition de "Juges généraux " régionaux : on en connait à Thessalonique, où le renom d'Harménopoulos fut peut-être à l'origine de l'institution nouvelle; à Lemnos sans doute; et sûrement en Morée.

Voilà l'essentiel de ce que l'on croit savoir : pour le détail, je me permets de renvoyer à mes précédents articles, où j'ai aussi donné les premiers résultats d'une enquête prosopographique partielle. Et voici maintenant ce qu'il y a lieu d'ajouter.

I. Autour du procès de corruption ${ }^{1}$ - - On sait qu'il nous est connu par Grégoras (XI, 3, 4: Bonn p. 536 - 538) : ému par les accusations de

1. Pour ce procès, la date de 1337, qui vient en fait de l'édition Bonn de Grégoras, a été donnée par L. Petit et par moi - même, et unanimement reprise. Mais un examen de la chronologie du livre XI de Grégoras, auquel m'ont conduit d'autres recherches ( cf. L'émirat d'Aydin, Byzance et l'Occident, chap. VI), m'amène à me demander maintenant si ce ne serait pas plutôt en 1336 qu'il faudrait placer le procès, malgré la mention toute voisine, chez Grégoras, d'éclipses communément placées en 1337. Je ne traiterai cependant pas ici cette question, qui ne trouvera de réponse ysûre (sauf documents nouveaux) que lorsque sera fixée la chronologie de Grégoras XI. Je signale seulement l'intérêt de l'acte synodal M.M. I no 77, p. 174 sq. : une pièce qui y est insérée, et qui est datée du 24 Octobre indiction $5(=1336)$, mentionne le juge général des Grecs Glavas (p. 177), et me paraît donc plutôt favorable à la date de 1337 . 
vénalité portées de tous côtés contre des juges qui avaient cependant prêté par écrit de terribles serments, l'empereur préside avec le patriarche, à Sainte-Sophie, un tribunal $(\beta \tilde{n} \mu \alpha)$ composé d'évêques et de prêtres - car il s'agit d'une affaire de violation de serment-devant lequel tous les plaignants sont invités à déposer ; les dépositions furent si nombreuses et accablantes, qu'à l'exception d'un seul reconnu innocent (Matarangos), les trois autres Juges généraux, " pour éviter la colère populaire"), se condamnèrent eux-mêmes à rendre les sommes qu'ils avaient touchées et à se démettre de toute fonction judiciaire; l'évêque (Joseph d'Aprô), en plus, se démit du sacerdoce; Andronic III ordonna en outre qu'ils fussent chassés de Constantinople.

Deux pièces sont venues récemment enrichir ce dossier. La première est un texte édité pour la première fois par G. Théocharidès, d'après un manuscrit autrefois conservé au gymnase grec d'Andrino-

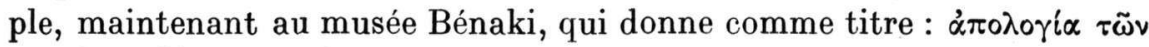

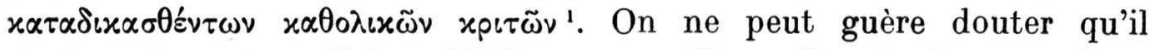
s'agisse de notre affaire. L'éditeur considère, d'après les premières lignes, que ce plaidoyer fut écrit après que les bannis furent rentrés à Constantinople; cela n'est pas certain, car voici la traduction : “ Ayant tant et si cruellement souffert d'une sentence injuste et portée contrairement aux lois, qui nous a infligé ce que les lois réservent aux

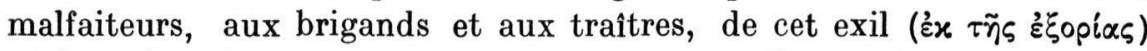
où les saints juges nous ont envoyés en nous livrant de leurs propres mains, du coeur même du sanctuaire, aux bourreaux, nous venons auprès de l'empereur, nous réfugier au port de ta philanthropie et de

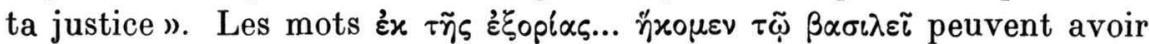
un sens figuré, et n'impliquent pas que les bannis soient de retour dans la capitale: si d'ailleurs ils avaient déjà obtenu leur pardon, on verrait mal le sens de cette longue apologie, qui s'adresse dans sa première partie à l'empereur et dans la seconde au patriarche (les deux présidents, nous l'avons vu, du tribunal ecclésiastique réuni à SainteSophie), et qui tourne autour de deux thèmes, celui de l'illégalité de la sentence de bannissement portée par des juges d'Église (mais Grégoras, lui, l'attribue bien à l'empereur!), et celui de l'injustice qu'il y a

1. G. J. Thé och a ridè s, " Die Apologie der verurteilten höchsten Richter der Römer ", Byz. Zeitschr., 56, 1963, p. 69-100: les pp. $69-79$ reprennent ce que l'on savait déjà sur les Juges généraux; le texte grec et une traduction allemande libre, sans commentaire, sont donnés aux pp. 79-99, et quelques (Schlussbemerkungen") présentées à la dernière page. 
à faire porter aux condamnés le poids de fautes commises par des employés. Je ne crois pas non plus, malgré G. Théocharidès, que le rédacteur de ce plaidoyer soit nécessairement un homme d'Église, et dans ce cas probablement Grégoire Kleidas : le ressentiment et l'amertume qu'il ne dissimule guère envers le patriarche et les juges ecclésiastiques feraient plutôt penser à un laïc, donc à Glavas. Bref l'étude détaillée de ce long texte, en comparaison avec le récit de Grégoras et avec le peu que nous savons par ailleurs ', reste à faire.

La seconde pièce, déjà publiée ${ }^{2}$ mais passée inaperçue, a été bien mise en valeur par I. Ševčenko ${ }^{3}$. Il s'agit d'une lettre adressée à Andronic III par un Léon prôtasèkrètis (l'hypothèse de Ševčenko qu'il s'agit de Léon Bardalès me parait la plus vraisemblable), au lendemain du jugement rendu par ( un tribunal terrible» contre " les malheureux juges ». L'auteur ne se plaint pas du sort qui, dans ce jugement, lui a été fait: il est libre, dans son pays, et non banni (donc les juges condamnés ont été bannis); il est désormais délivré des tracas de toutes

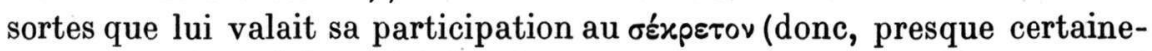
ment, au tribunal impérial des Juges généraux); mais ses biens ont été

1. J'ai déjà, par exemple, signalé (“ Le juge général...), p. 308, n. 3) l'intérêt de la sentence synodale d'Avril 1342 éditée M.M. I, no 99, p. 226 - 227. Elle concerne Michel Kabasilas, ami d'enfance et parent par alliance de Joseph métropolite d'Aprô. Nous y apprenons que lorsque celui - ci devint Juge général,

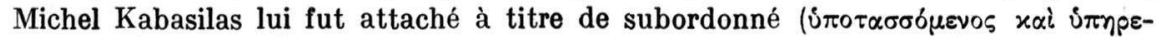

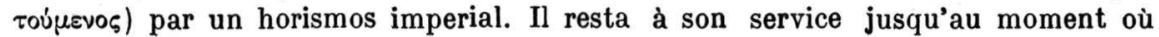
Joseph, lors du procès de corruptton, “démissionna de l'épiscopat et de sa charge de jugen: confirmation précieuse du récit de Grégoras. Quelques années plus tard, en 1342 sans doute, Michel Kabasilas voulut entrer dans les ordres, mais certains s'y opposèrent, rappelant qu'il avait été au service de Joseph d'Aprô et soup-

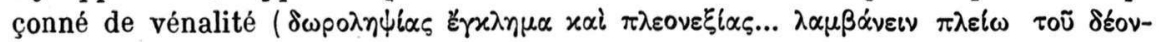

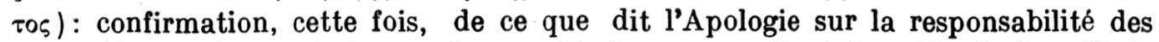
employés des Juges généraux. On procéda à une enquête à la suite de laquelle le Synode prononce qu'il n'y a pas obstacle à l'entrée de Michel Kabasilas dans les ordres, avec des considérants curieux : qu'il ait touché peu ou beaucoup à l'occasion de ses fonctions auprès d'un juge n'est pas un empêchement, est-il dit, car il n'était pas lui-même juge.-Ce texte n'implique naturellement pas qu'en Avril 1342, Joseph d'Aprô soit revenu d'exil ou ait été rétabli dans ses droits (contra Thé oc h a ri dè s, op. cit., p. 79).

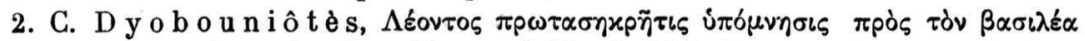

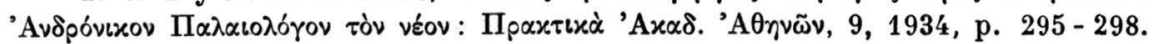

3. I. Š e v દ̌ e n o, "Léon Bardalès et les Juges généraux, ou la corruption des incorruptibles \#, Byzantion, 19, 1949 (Actes du VIIe Congrès des Études byz., I ), p. $247-259$. 
entièrement confisqués; or s'il est juste de confisquer les biens de juges convaincus de corruption (les Juges généraux se sont en effet condamnés eux-mêmes à rembourser ce qu'ils avaient touché) et qui ont violé leur serment (c'est bien l'accusation principale portée contre eux, et qui a motivé la réunion d'un tribunal ecclésiastique à Sainte-Sophie), il n'est en revanche pas juste que soient compris dans la confiscation les biens de tiers détenus à titre de dépôt ou de prêt. On voit qu'il n'est guère douteux que l'affaire dont il s'agit ici soit, comme l'a bien vu Ševčenko, le procès même qui nous occupe. Mais il est certain que le prôtasèkrètis Léon n'était pas l'un des quatre Juges généraux qui en furent les vedettes, puisque de ceux - ci nous connaissons les noms. Il faut donc admettre, malgré le silence sur ce point de Grégoras, que d'autres personnes y furent impliquées, et condamnées.

De cela, Ševčenko a tiré des conclusions qui vont loin. D’abord, que les Juges généraux créés par Andronic III, “ tout en jouissant de prérogatives très étendues et formant la plus haute instance du pouvoir judiciaire, ne se sont pas entièrement substitués au suprême degré de la juridiction ancienne ), autrement dit que le prôtasèkrètis a conservé ses attributions judiciaires. Puis, établissant une équation entre " tri-

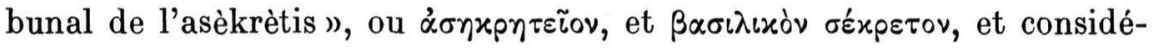

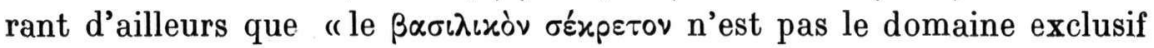
des Juges généraux ", il pense que le second est plus ou moins issu du premier et n'en est pas, au moins à l'origine, complètement distinct : “ L'institution des Juges généraux, création nouvelle, reposerait sur un

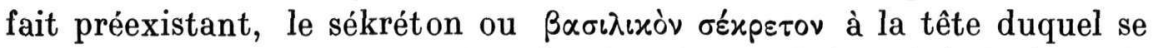
trouverait jusque là un prôtasèkrètis. Après 1329, celui-ci siégerait avec les Juges généraux dans son tribunal envahi par eux. Il aurait perdu sa place prépondérante, mais pas suffisamment pour ne pas être atteint, pour ainsi dire à droits égaux, par la sévère condamnation de 1337. Peut-être Léon Bardalès serait-il le dernier des prôtasèkrètis effectifs? ') Ces hypothèses ingénieuses, développées avec le talent qu'on connaît à M. Ševčenko, ne me paraissent nullement convaincantes. Sans doute, et c'est de bon sens, la création du tribunal suprême des Juges généraux ne s'est pas faite ex nihilo, et n'a pas fait disparaître toutes les autres instances judiciaires. Mais dans l'état actuel de nos connaissances, tout nous interdit de penser que le basilikon sékréton des Juges généraux soit sorti d'un tribunal du prôtasèkrètis, et que d'une manière quelconque l'asèkrèteion subsiste dans le sékréton. J'ignore comment et pourquoi le vieux Léon Bardalès, à quatre-vingts ans, a été impliqué dans le procès des Juges généraux, et a vu ses biens confis- 
qués : il ne nous le dit pas. J'admets qu'à côté et au-dessous des Juges généraux, le tribunal impérial devait comprendre un nombreux personnel, de rangs divers. Nous avons déjà vu, dans des fonctions relativement modestes, mais tout de même nommé par horismos de l'empereur, Michel Kabasilas ${ }^{1}$. Léon Bardalès, à un niveau sans doute plus élevé, non de simple exécutant, est un autre exemple. Je n'oserais aller plus loin pour le moment.

II. Syméon, Juge général en 1342? - I. Ševčenko s'était avec raison demandé ${ }^{2}$ si le " nomophylax » destinataire d'une lettre de Palamas de 1343/4 (Paris. Coislin 99, fol. 110 R) ne serait pas Harménopoulos. J. Meyendorff ${ }^{3}$ a montré que cette hypothèse doit être abandonnée, car le nom du nomophylax correspondant de Palamas est connu par d'autres manuscrits: Syméon. Mais à son tour il se demande si ce

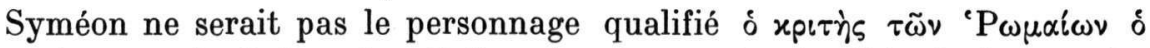

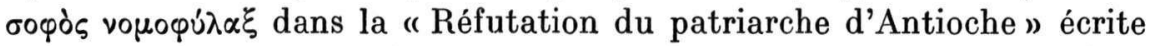
par Palamas: en Juin 1342, ce “Juge des Grecs» fut envoyé par le patriarche pour convoquer Palamas au patriarcat ${ }^{5}$. Dans ce cas, il faudrait introduire Syméon dans la série des Juges généraux des Grecs, en 1342 , et peut-être en $1343 / 4$.

III. La date de nomination d'Antoine de Larissa. - G. Théocharidès a publié des documents inédits des archives de Vatopédi, et notamment ${ }^{6}$ la copie d'un acte de donation établi par Arsénios Tzamplakôn en Mai 1356. Or cette copie, pour la date de laquelle le terminus post est donc

1. Cf. ci-dessus, page 32, note 1. Je rappelle que dans l'acte synodal d'Avril 1342, les fonctions de Michel Kabasilas auprès de Joseph d'Aprô sont ainsi définies :

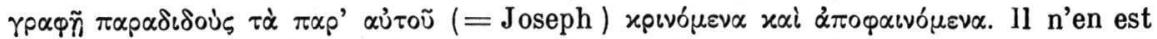
pas moins compromis dans les remous du grand procès, et fortement soupçonné, en 1342 encore. Le prôtasèkrètis Léon, qui devait avoir un rôle plus important, a fort bien pu être englobé dans la condamnation des Juges généraux, sans être toutefois frappé de cette mesure de bannissement qui semble bien le principal sujet de l'Apologie dont nous avons parlé plus haut.

$\therefore$. I. Š e v̌̌ e n k, “ Nicolaus Cabasilas' correspondence and the treatment of late Byzantine literary texts ", Byz. Zeitschr., 47, 1954, p. $49-59$ : cf. p. 51.

3. J. Me yendo r f f, Introduction à l'étude de Grégoire Palamas, Paris, 1959, p. 367

4. Il n'est guère douteux qu'il s'agisse d'un Juge général des Grecs.

5. Sur cet épisode, J. M e y e n d o r f f, op. cit., p. 102.

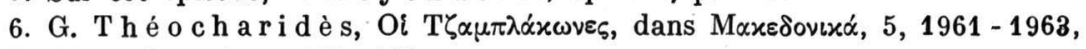
p. $125-183$ : cf. no 2 , p. $133-138$. 
Mai 1356, est authentifiée par la signature du métropolite hypertime de Larissa, exarque de la Thessalie Seconde et de toute l'Hellade, Antoine : il ne porte pas le titre de Juge général des Grecs, qu'il n'eût sûrement pas omis. Or on connaissait déjà, de cet Antoine, un acte de Juin 1340, où en effet il n'est pas dit Juge général; un acte de Juin 1359 et un de Mai 1362, où il porte ce titre ${ }^{1}$. On en conclut que la copie authentique de l'acte de donation de Tzamplakôn a été dressée entre Mai 1356 au plus tôt (date de l'original), et Juin 1359 au plus tard (date à laquelle Antoine est Juge général); et que la nomination d'Antoine comme Juge général des Grecs s'est faite entre ces deux dates.

IV. Andronic Oinaiôtès, Juge général en 1369 - 13\%1. - R. Loenertz a publié une lettre écrite par Dèmètrios Cydonès à un Juge général des Grecs nommé Andronic Oinaiôtès, lettre qu'il date de 1369-1371. Dans ce cas, ce ne saurait être le même que l'Oinéôtès de prénom inconnu qui porte le même titre en Août 1400, et forme collège avec Jean Syropoulos et Chrysoképhalos ${ }^{3}$ : nous savons en effet que ce trio avait été précédé d'un collège comprenant Macaire de Nicomédie (attesté comme Juge général en 1393), Alousianos et Kaballaropoulos 4 . Donc on est conduit à enregistrer deux Juges généraux des Grecs appartenant à la famille Oinaiôtès, Andronic en $1369-1371$, et un autre de prénom inconnu en Août 1400.

V. L'activité judiciaire à Thessalonique en 1373-1375. - Une série de mentions attestent l'existence de " Juges (généraux) de Thessalonique " depuis Harménopoulos et pendant trois quarts de siècle ${ }^{5}$. Mais cette époque est, dans l'histoire de la Macédoine, fort agitée. De très nombreux litiges, résultat des circonstances de la domination serbe, éclatèrent lorsque celle-ci s'effondra ou, du moins, recula. Nous en avons la preuve dans maints documents des archives de l'Athos, qui apportent de précieuses indications sur les institutions judiciaires, et meriteraient de ce point de vue une étude d'ensemble. Aussi longtemps que

1. P. Le merle, "Le Juge général... ", op. cit., p. 311.

2. R. Loenertz, Correspondance de Démétrius Cydonès, I (Studi e

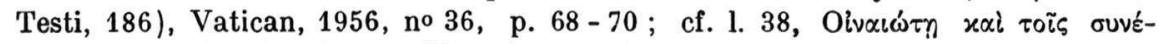

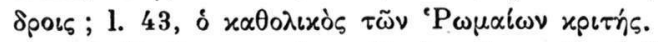

3. P. L e merle, " Recherches... Le tribunal impérial », op. cit., p. 382.

4. Ibid., p. 381 .

5. Je les ai énumérés dans les articles cités ci-dessus, page 29 , note 1 . 
celle-ci ne sera pas faite, et que d'ailleurs beaucoup de pièces demeureront inédites, de graves incertitudes subsisteront. Je me bornerai à signaler ici un certain nombre d'actes, pour la plupart récemment publiés, qui ont ou peuvent avoir un intérêt pour le problème des Juges généraux.

a) Les archives de Dochiariou conservent un acte de vente établi en Août 1373 par la grande domestikissa Anna Cantacuzène Palaiologina ${ }^{1}$. Il a été dressé, évidemment à Thessalonique, par Jean Sgouropoulos, sur l'invitation ( $\dot{\varepsilon} x \pi \rho \circ \tau \rho \pi \tilde{\eta} s)$ du prôtonotaire [Nicétas] Sôtèriôtès. Au verso, une clause additionnelle est relative à un droit que le couvent de Vatopédi renonce à faire valoir; puis il est spécifié que l'acte lui-même et cette clause ont été soumis au métropolite de Thessalonique, pour qu'il les confirme et garantisse. Viennent alors deux signatures: celle du métropolite de Thessalonique, Dorothée; et celle

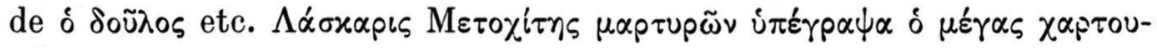
$\lambda$ ápıos.

b) Or F. Dölger a publié ${ }^{2}$ un acte des archives de Lavra, de Février 1374, par lequel Alexis Comnène Masgidas met fin, moyennant versement d'une somme forfaitaire, à un conflit qui l'opposait au couvent. Après les signatures de Masgidas et de sa partie, et asant celle de Jean Sgouropoulos qui a dressé l'acte, se placent trois signatures,

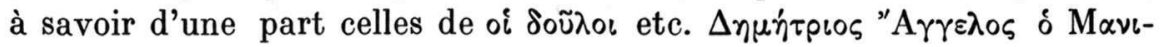

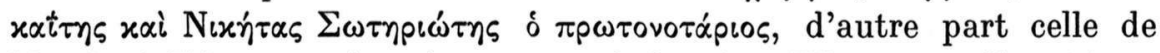

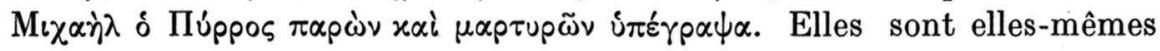

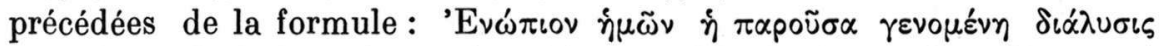

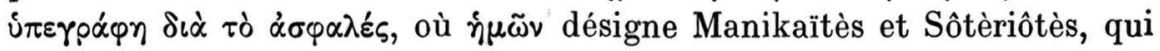
ne sont pas des témoins, la seule signature de témoin étant apparemment celle de Michel Pyrrhos. Qui sont Manikaïtès et Sôtèriôtès? F. Dölger considère que celui-ci est prôtonotaire du thème ${ }^{3}$, et Manikaïtès juge du thème, puisque la correspondance de Cydonès le désigne comme juge $^{4}$. Disons tout de suite un mot de ce dernier. Il est exact qu'une lettre de Cydonès adressée à un Manikaïtès, maintenant connue par

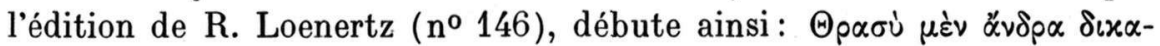

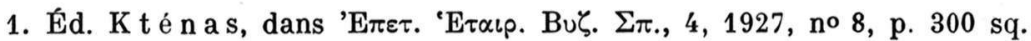

2. F. Dölg e r, Aus den Schatzkammern..., no 113.

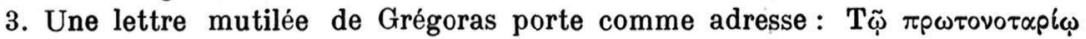

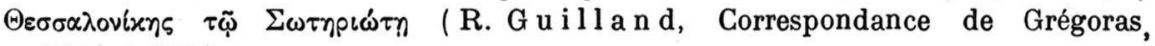
p. 117 et 377$).$

4. F. Dölger ne pouvait encore connaître que l'indication imprécise de C a m melli, Correspondance de Cydonès, p. 210. 


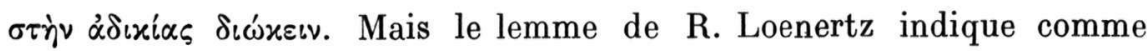
destinataire : "Demetrio Ducopulo Manicaitae, judici, Thessalonicam ", comme d'ailleurs pour les lettres $\mathrm{n}^{0} 116$ (de 1372-1373?), 144 et 148. Or le signataire de l'acte de Lavra de Février 1374 est Angélos Manikaïtès. D'où vient Doukopoulos? D'une part d'une lettre, notablement plus ancienne, de Nicolas Kabasilas ${ }^{1}$; d'autre part d'un acte encore inédit des archives du couvent athonite du Pantocrator ${ }^{2}$, par lequel, en Mars 1368, Marie Laskarina vend un terrain au grand stratopédarque Alexis, en présence du grand papias Dèmètrios Doukas Kabasilas, de Dèmètrios Doukopoulos Manikaïtès et d'Alexis Hyalôn Laskaris (l'acte est dressé par le diacre Nicolas Synadènos, sakelliou de l'Eglise de Thessalonique). En attendant de nouveaux documents, on doit donc admettre qu'il faut distinguer: 1. Dèmètrios Doukopoulos Manikaïtès, qui est probablement le correspondant de Nicolas Kabasilas, et sûrement le signataire de l'acte de Mars 1368; 2. Dèmètrios Angélos Manikaïtès, qui est le signataire de l'acte de Lavra de Février 1374 et d'autres pièces que nous allons voir, et qui pourrait bien être-je laisse au R. P. Loenertz le soin d'en décider-le correspondant de Cydonès.

c) Il faut revenir sur un document que j'avais déjà eu l'occasion de signaler ${ }^{3}$, l'acte de Chilandari no 154, établi en Avril 1374. La partie Isaris, en conflit avec Chilandari, était sur le point de comparaitre

1. Édition par P. En épéki dès, “ Der Briefwechsel des Mystikers Nikolaos Kabasilas ", Byz. Zeitschr., 46, 1953, p. $18-46$ : cf. no 16, p. $42-43$.

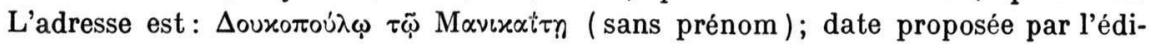
teur : "probablement avant 1345 ». Mais sur les difficultés que présente la chronologie de Nicolas Kabasilas en général, cf. I. Š e č enko, Byz. Zeitschr., 47, 1954, p. 54 sq.; R. L o e nertz, "Chronologie de Nicolas Cabasilas 1345-1354", Orient. Christ. Period., 21, 1955, p. $205-231$. Nicolas Kabasilas a-t-il refusé d'être Juge général, ou bien démissionné de cette charge? Comme l'a rappelé R. Loenertz (" Pour la chronologie des œuvres de Joseph Bryennios », Rev. Ét. Byz., 7, 1949, p. 17), Manuel II, dans une lettre qu'il écrit d'Asie Mineure à Kabasilas en 1391, lui dit qu'à son retour il le plaisantera sur le fait que sa

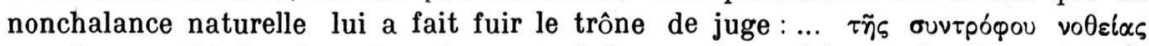

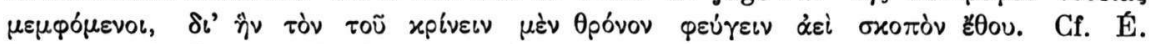
Legrand, Lettres de l'empereur Manuel Paléologue, Paris, 1893, p. 20-21, l. $39-44$.

2. La photographie, provenant de la mission Millet, se trouve entre les mains de V. Laurent, qui l'a communiquée à $\mathrm{R}$. L o e $\mathrm{n}$ e $\mathrm{rtz}$ : cf. Correspondance de Démétrius Cydonès, II (Studi e Testi, 208), p. 14, note.

3. "Recherches... Le tribunal impérial ", op. cit., p. 378. On voudra bien supprimer là, 1. $12-13$, les mots : “découragées... intéressante ». 


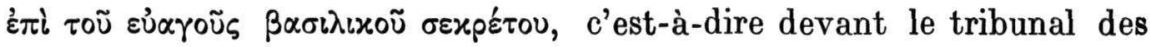
Juges généraux (des Grecs, à Constantinople?). En fin de compte, elle y renonce, et préfère un accord amiable : il fait l'objet du présent acte,

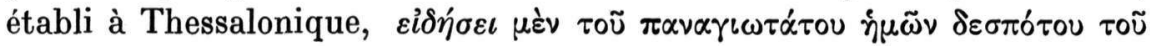

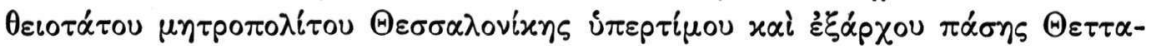

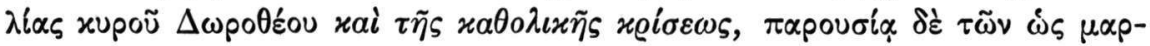

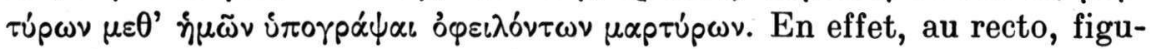
rent les signatures annoncées de la partie Isaris et des témoins. Mais au verso figurent : d'abord la signature du métropolite Dorothée, sans

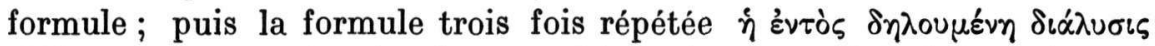

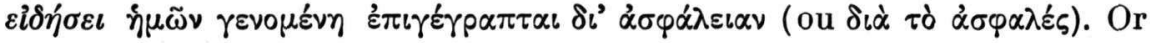
la première fois cette formule est suivie des signatures (incorrectement

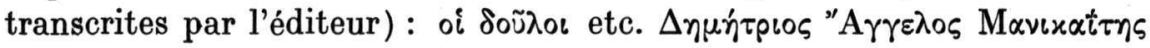

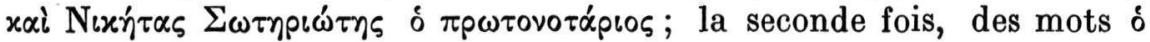

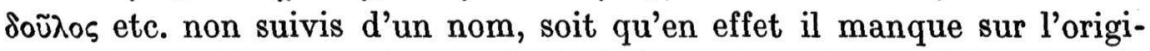
nal, soit que l'éditeur n'ait pu le déchiffrer; la troisième fois enfin, de

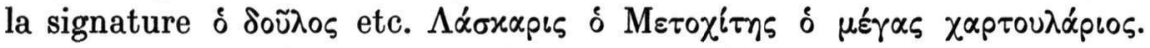
Donc d'une part le texte mentionne une $x \alpha \theta 0 \lambda \iota x \dot{\eta}$ xpícเs qui ne peut pas être le tribunal des Juges généraux des Grecs à Constantinople, devant qui l'affaire n'est sûrement pas venue, mais celui des Juges généraux de Thessalonique, où l'acte est en effet établi. D'autre part, si les signatures du recto sont bien celles de la partie et des témoins, on attend que celles du verso soient celles des personnages qu'annonce

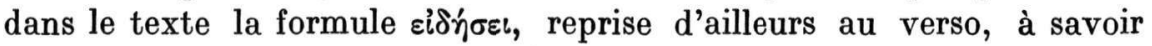

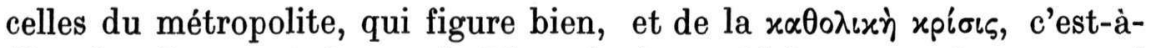
dire des Juges généraux de Thessalonique. Mais aucun de ceux qui signent effectivement au verso après le métropolite ne se désigne comme

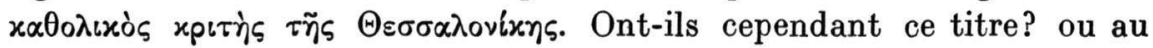
moins certains d'entre eux, Manikaïtès et Sôtèriôtès?

d) L'acte de Kutlumus no 33 est un jugement rendu à Serrès, en Août 1375, par un tribunal composé du métropolite de Serrès Theodosije, du képhali Tarchaneiôtès, et de plusieurs dignitaires ecclésiastiques et laíques de Serrès. Avant de juger, ils avaient consulté à Thessalonique plusieurs personnes compétentes en matière judiciaire: le métropolite hypertime et exarque de toute la Thessalie (c'est donc Dorothée); son grand chartophylax (on sait par une autre pièce du même dossier

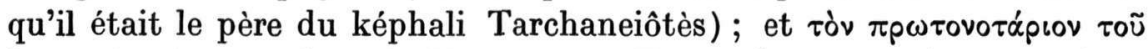

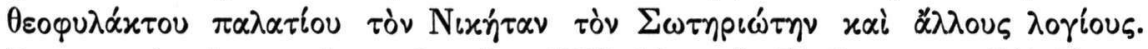
Deux mois plus tard, en Octobre 1375 (Acte de Kutlumus no 34), l'acte rendu à Serrès a été présenté pour confirmation à deux personnages 


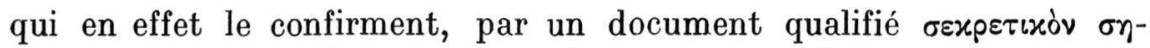
$\mu \varepsilon i \omega \mu \alpha$, et revêtu de leurs deux signatures, mutilées sur la pièce de Kutlumus, mais qu'on peut maintenant restituer avec certitude: $\mathrm{O}_{i}$

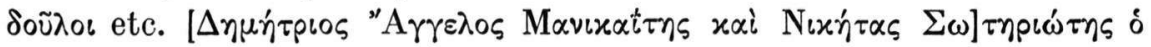
$\pi \rho \omega \tau$ ovorápios. Pas plus que dans les documents précédents, ils ne se disent “juges généraux de Thessalonique ». Mais: 1) ils nomment $\sigma \varepsilon-$

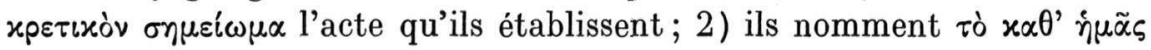

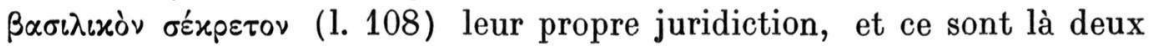
très fortes raisons de penser qu'il s'agit du tribunal des Juges généraux ; 3) des Grecs à Constantinople, ou bien de Thessalonique? la vraisemblance et la comparaison avec d'autres cas analogues suggèrent Thessalonique, et tout doute est levé par le titre que le copiste, très peu d'années après l'établissement de l'original (car cette copie est authentifiée par l'évêque d'Hiérissos et Athos Isaac, aux alentours de 1380),

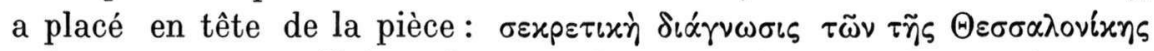
$x \propto \theta 0 \lambda$ เx $\tilde{\omega}_{\nu} x p \iota \tau \tilde{\omega} \nu^{1}$. Il faut done conclure que c'est bien la fonction que remplissaient, à cette date, Manikaïtès et Sôtèriôtès, bien qu'il soit difficile d'expliquer pourquoi ils n'en portent pas le titre.

e) G. Théocharidès a récemment publié plusieurs documents inédits des archives de Vatopédi ${ }^{2}$ : un chrysobulle d'Andronic II, de Juin 1324, en faveur de Théodore Sarantènos et du couvent du Prodrome qu'il a fondé à Béroia; le testament de Sarantènos, d'Octobre 1325, énumérant tous les biens dont il dote son couvent; un acte du domes-

1. Je corrige ici ce que j'ai dit dans mon édition des Actes de Kutlumus, ainsi que dans "Le Juge général...", p. 307 n. 1, et dans " Recherches... Le tribunal impérial », p. 379. N'ayant pu alors compléter les signatures, et trompé

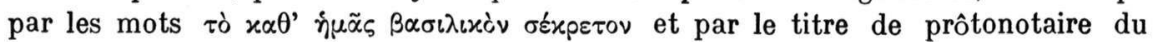
Palais porté par Sôtèriôtès, j'avais cru que la pièce émanait des bureaux impériaux de Constantinople, et que l'attribution aux Juges généraux de Thessalonique, que faisait le copiste, était une erreur de celui - ci. Notons à ce propos l'intérêt

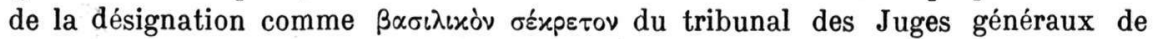
Thessalonique: elle est nouvelle, je crois, et laisse planer un doute sur l'identité du tribunal dont il est question au début de l'acte de Chilandari no 154 , examiné ci-dessus; il pourrait s'agir, là aussi, de Thessalonique.

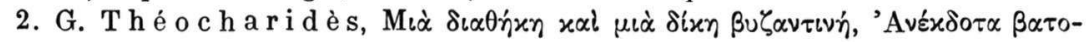

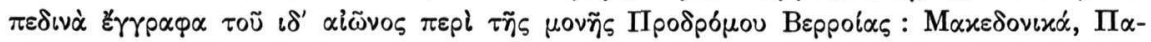

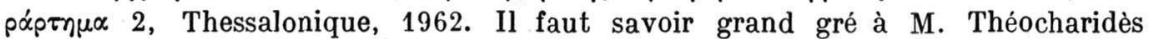
d'avoir fait connaitre des pièces si importantes. Je dois avouer que souvent je ne suis pas d'accord avec son commentaire, mais il n'est pas utile d'énumérer ici ces divergences : je me borne à tirer de ces documents nouveaux ce qui intéresse mon sujet. 
tique des thèmes Constantin Makrènos, de Mai 1338, en faveur de Vatopédi, devenu entre temps propriétaire du Prodrome de Béroia. Passons sur les circonstances dans lesquelles Vatopédi a mis la main sur le couvent : en tout cas des descendants de Sarantènos contestèrent obstinément qu'il en fût bien le maitre. L'affaire fut jugée, en Juin 1375 , en faveur de Vatopédi, comme nous l'apprennent les deux derniers documents, qui sont ceux qui nous intéressent. C'est d'abord un long sèmeiôma très détaillé, signé par le métropolite de Thessalonique

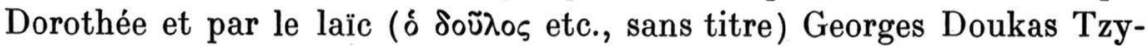
kandèlès, qu'un horismos de l'empereur (Jean V) avait chargés de " se

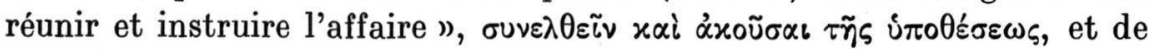

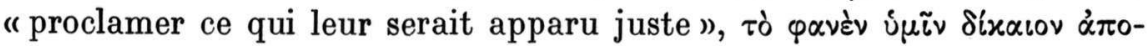

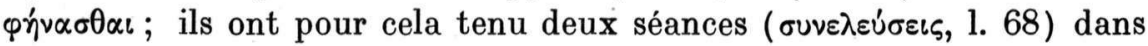
la métropole de Thessalonique ${ }^{1}$. C'est enfin une courte pièce (neuf lignes), signée par Dèmètrios Angélos Manikaïtès et le prôtonotaire Nicétas Sôtèriôtès dans la même forme que tous les actes examinés cidessus : ils déclarent aussi qu'un horismos impérial leur a enjoint de se réunir ( $\sigma u v \varepsilon \lambda \theta \varepsilon i ̃ v)$ avec le métropolite de Thessalonique pour juger l'affaire, qu'ils ont attentivement au cours des deux sessions écouté les dépositions des parties et examiné leurs pièces, et enfin décidé en faveur

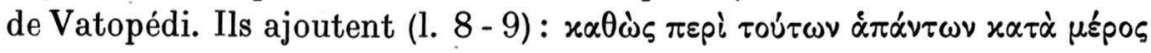

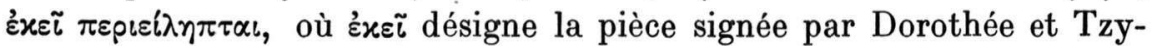
kandèlès, en effet très détaillée, à laquelle celle-ci ne fait qu'apporter la confirmation de deux autres juges. Ces pièces telles que nous les connaissons présentent certaines difficultés diplomatiques, que lèverait peut-être l'examen des originaux à Vatopédi. Ce qui nous importe est que l'empereur, pour juger cette affaire, a saisi un tribunal composé de quatre personnes, le métropolite de Thessalonique et trois laïcs. De ce chiffre de quatre, G. Théocharidès a conclu qu'il s'agit de quatre Juges généraux : mais cet argument ne saurait valoir que pour les débuts de l'institution car, si je ne me trompe, le seul collège de quatre Juges généraux actuellement connu est celui qui fut créé en 1329. Par d'autres considérations, il est conduit à penser que, dans ce tribunal, le métro-

1. Parmi les pièces présentées par Vatopédi à l'appui de ses droits figure

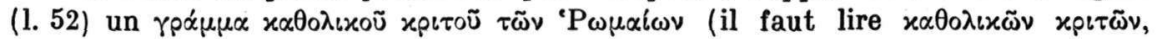
comme le confirment les 1.72 et 82 sq.), qui déjà prononçait en faveur de Vatopédi contre les revendications d'un neveu de Sarantènos: décision et acte se placent sûrement avant l'occupation serbe; un extrait de cet acte des Juges généraux est inséré 1.82 sq. 
polite Dorothée et Georges Doukas Tzykandèlès sont des Juges généraux de Thessalonique, tandis que Manikaïtès et Sôtèriôtès viendraient de Constantinople et seraient donc des Juges généraux des Romains: je suis obligé de dire qu'à mon avis rien ne justifie cette conclusion, et qu'elle est même exactement à l'opposé du vrai, si nous avons bien établi ci-dessus que Manikaïtès et Sôtèriôtès sont des Juges généraux de Thessalonique. Il n'y a pas lieu de trop s'étonner que quatre juges ayant siégé ensemble signent deux par deux : comme dans d'autres cas, cela peut s'expliquer par une circonstance fortuite (l'acte, souvent fort long, n'était pas dressé sur le champ, et il n'était pas toujours facile de réunir à nouveau, pour le signer, ceux qui avaient siégé ensemble); on peut aussi dans notre cas préférer penser que les deux Juges généraux de Thessalonique, pour une raison quelconque, ont jugé bon d'établir un acte particulier. En tout cas rien ne nous permet actuellement de dire que le métropolite Dorothée et Tzykandèlès étaient Juges généraux de Thessalonique. L'empereur, pour chaque affaire, peut toujours composer ou compléter le tribunal comme il l'entend. Le métropolite, dans ce cas comme dans d'autres que nous avons précédemment vus, est là es qualité, parce qu'une des parties est un couvent. De Tzykandèlès je ne saurais pour le moment rien dire, sinon qu'il tient peut-être une place comparable à celle que dans d'autres pièces nous avons vu tenue par le grand chartoularios Laskaris Métochite (et Michel Pyrrhos?). Manikaitès et Sôtèriotès sont Juges généraux de Thessalonique.

VI. A propos du Juge général Alousianos. - J'ai autrefois ${ }^{1}$ signalé l'existence d'un collège de Juges généraux des Grecs comprenant le métropolite de Nicomédie Macaire (connu d'autre part comme tel en Mars 1393), Kaballaropoulos (Constantin?), et un Alousianos dont je ne savais alors rien de plus. R. Lœnertz a depuis édité quatre lettres de Cydonès adressées sûrement ou probablement à Thomas Doukas Alousianos 2, qui est notre Juge général. C'est ce qu'établit, si du moins il en est bien le destinataire, la lettre no 440, datée par l'éditeur d'environ 1391 - 1393 : les lignes 61 - 85 sont intéressantes pour le fonction-

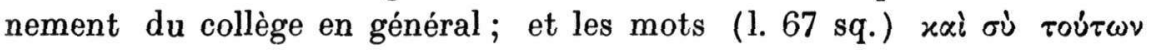

1. "Recherches... Le tribunal impérial ", op. cit., p. 381.

2. R. L o e n e r tz, Correspondance de Cydonès, op. cit., II, lettres no 208 (cf. note: Alousianos présent à la signature du traité entre Jean $\mathrm{V}$ et Gènes en 1382 ; honoré de la citoyenneté vénitienne en 1383), 246, 319, 440. 


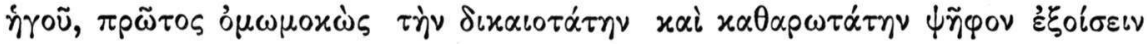
ont fait penser à R. Lœnertz qu'Alousianos devait en être le président.

VII. Un collège de Juges généraux dans le Voyage de Mazaris aux Enfers?-G. Théocharidès a rappelé ${ }^{1}$ que cet ouvrage, dont on considère que la rédaction se place en 1414-1415, contient une série de noms de personnages contemporains dissimulés sous des jeux de mots

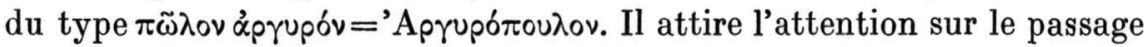

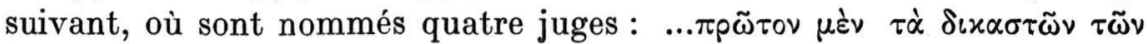

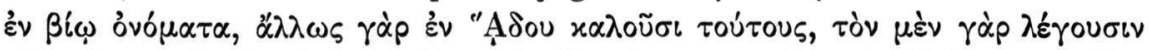

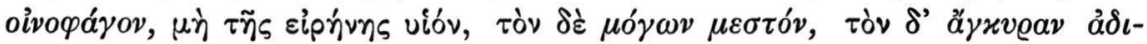

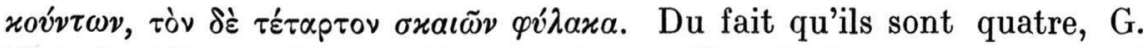
Théocharidès considère qu'il s'agit d'un collège de Juges généraux : j'ai déjà dit que cela ne me paraît pas être une preuve suffisante. Et il

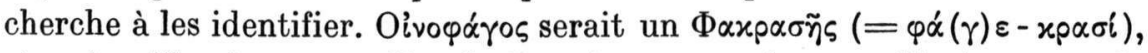
et puisqu'il n'est pas fils de la paix, ce serait un militaire: non le prôtostratôr bien connu Georges Phakrasès, hors d'âge en 1415, mais peut-être un Phakrasès connu comme déphensôr en 1421, quoiqu'il soit

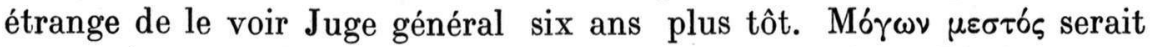

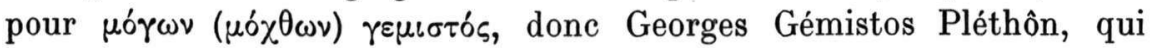
aurait été Juge général de Morée. Après ces deux laïcs, il faut deux

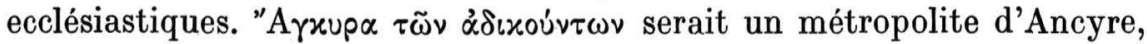
d'ailleurs inconnu. $\Sigma x \alpha \iota \tilde{\omega} v$ $\varphi \dot{\lambda} \alpha \xi_{\xi}$ enfin serait un skeuophylax de la Grande Église, en même temps nomophylax, parce que $\sigma x \alpha$ เòs $=\alpha \dot{\sigma} \tau \eta$ pós, et que "dura lex, sed lex»; et ce serait Jean Syropoulos ${ }^{2}$. Il saute aux yeux que ce mélange de Juges généraux des Grecs (de Constantinople), et de Morée, ne fait en tout cas pas un collège. Pour le reste, tout ce que l'on peut dire est qu'il s'agit d'une ingénieuse hypothèse, qui attend un commencement de preuve.

VIII. Sylvestre Syropoulos, Juge général des Grecs. - En 1437, alors que l'empereur et le patriarche sont sur le point de quitter Constantinople pour l'Italie pour se rendre au concile, beaucoup de dignitaires de l'Église, désignés pour les accompagner, cherchent à se récuser. De

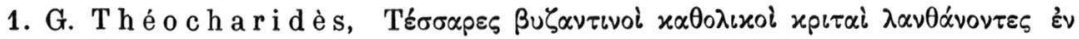

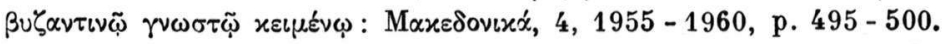

2. Sur le collège composé de Jean Syropoulos, grand skeuophylax de Sainte Sophie, Chrysoképhalos et Oinéôtès, attesté en Août 1400, cf. Lemerle, "Recherches... Le tribunal impérial ", op. cit.,'. p. 382. 
ce nombre est Sylvestre Syropoulos, qui demande au mésazôn Notaras d'intervenir auprès de l'empereur. Notaras s'acquitte de cette mission, d'ailleurs en vain comme l'on sait, et pour persuader l'empereur de

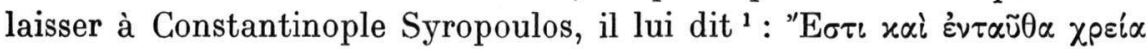

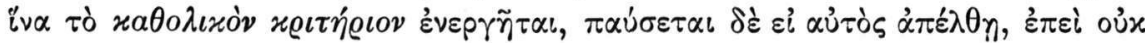

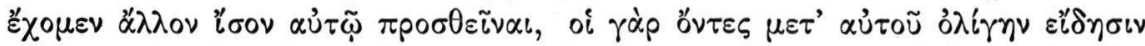

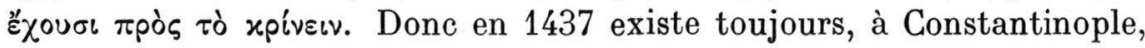
le tribunal des Juges généraux des Grecs (katholikon kritèrion), dont le bon fonctionnement est tenu pour indispensable. Sylvestre Syropoulos en est le principal membre, peut-être le président, le seul en tout cas à pouvoir en assurer la bonne marche, car ses collègues, les autres Juges

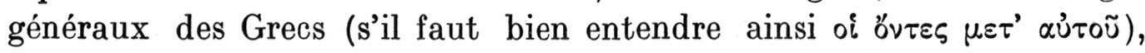
au nombre donc d'au moins deux, peut-être des laïcs, ont peu de compétence en matière de justice.

IX. Francoulis Servopoulos, Juge général des Grecs, et Pléthon, Juge général de Morée? - Dans le second volume de sa grande histoire du Despotat grec de Morée, D. Zakythinos a traité des Juges généraux de Morée $^{2}$. Il donne des indications plus précises et plus exactes que les miennes ${ }^{3}$ sur Francoulis Servopoulos: une lettre du roi de Naples, de Juin 1454, le qualifie " olim imperialis cancellarius ac judex Romæorum generalis " ; quant à la lettre latine écrite en Décembre 1455, au roi de France Charles VII, par le despote Dèmètrios Paléologue ${ }^{4}$, elle s'exprime ainsi : «nobilum (sic) virum nostrum Francalium (sic) Servopulum, fratrum olim meorum imperatorum cancellarium et Romeorum judicem generalem ». Il est donc certain que Francoulis Servopoulos a été, non pas Juge général de Morée, comme je l'avais cru, mais Juge général des Grecs, à Constantinople, sous les deux derniers empereurs, Jean VIII et Constantin XII, en effet frères du despote Dèmètrios; et D. Zakythinos a raison de penser qu'après la chute de la capitale il dut chercher refuge dans le Despotat. Je me demande s'il n'avait pas, dans

1. Vera historia unionis non verae..., éd. R. Creyghton, 1660, p. 53.

2. D. A. Z a kythinos, Le Despotat grec de Morée, II, Vie et institutions, Athènes, 1953, p. $129-131$ - Je signale que, p. 130, sont citées les éditions de la notice et de la signature de Nicolas Boullôtès Agallôn, Juge général de Morée en 1447 ( P. Le merle, "Le Juge général... ", op. cit., p. 315), et qu'un fac-simile en est donné.

3. P. Le merle, “Le Juge général... », op. cit., p. 316.

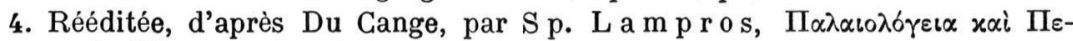

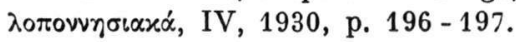


sa double charge (“ chancelier » et Juge général), succédé à Scholarios, qu'une notice ${ }^{1}$ qualifie de $x \alpha \theta 0 \lambda$ เxòs $\sigma \varepsilon x p \varepsilon \tau \dot{p}$ pıos de Jean VIII et Juge général des Grecs. D. Zakythinos pense d'autre part que Georges Gémistos Pléthon a été Juge général de Morée (on a vu plus haut que G. Théocharidès, d'après Mazaris, forme la même hypothèse), parce que

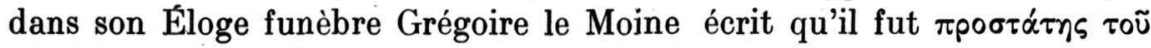

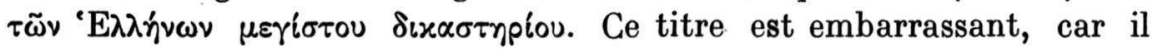
suggère plutôt celui de Juge général des Grecs (et même la présidence du tribunal, à Constantinople) que de Juge général de Morée. Le dernier biographe de Pléthon, F. Masai ${ }^{2}$, admet cependant, à la suite de D. Zakythinos, que Pléthon fut Juge général de Morée, et il parait penser que ce fut le poste que Manuel II lui confia lorsque, sous la pression de l'Église, il fut contraint de l'éloigner de Constantinople (done après 1405 , s'il est vrai qu'à cette date Pléthon professait encore dans la capitale). Mais ce n'est qu'une hypothèse, et la nomination d'un Juge général de Morée revenait-elle bien à Manuel II? Bref le cas de Pléthon ne me parait pas encore clair.

Paris, Septembre 1963.

Paul LEMERLE

P.S. Pour la chronologie d'Harménopoulos, cf. maintenant J. V e r p e a u x, dans Rev. Et. Byz., 21, 1963, p. 231 : “ La désignation d'H. comme Juge général - des Grecs ou de Thessalonique ? - est postérieure à 1359 et contemporaine du début du secont patriarcat de Philothée (1364-1376) ou de peu antérieure. ”

1. P. Le merl e, “ Le Juge général... », op. cit., p. 315.

2. F. M a s a i, Pléthon et le platonisme de Mistra, Paris, 1956, p. 63, n. 2. 